\title{
Randomised, prospective clinical trial of platelet-rich plasma injection in the management of severe dry eye
}

\author{
Marcel Y Avila, Angela M Igua, Amparo M Mora
}

Department of

Ophthalmology, Facultad de

Medicina, Universidad Nacional

de Colombia, Bogota, Colombia

\section{Correspondence to}

Dr Marcel Y Avila, Department of Ophthalmology, Facultad

de Medicina, Universidad Nacional de Colombia, Bogota, DC, Colombia; myavilac@unal. edu.co

Received 14 February 2018 Revised 19 May 2018 Accepted 4 June 2018 Published Online First 3 July 2018
A Check for updates

(c) Author(s) (or their employer(s)) 2019. No commercial re-use. See rights and permissions. Published by BMJ.

To cite: Avila MY, Igua AM, Mora AM. Br J Ophthalmol 2019:103:648-653.

\begin{abstract}
Background To evaluate the effectiveness of plateletrich plasma (PRP) injections in the treatment of severe dry eye.

Methods This prospective, intervention study included patients with severe dry eye who had been diagnosed with Sjogren syndrome. Patients were divided into two groups. The intervention group received PRP $(n=15)$ injections on days $0,30,60$ and 90 , as well as hyaluronic acid five times per day. The comparison group received hyaluronic acid $(n=15)$ five times per day. Subjects were measured at baseline and at 30,60 and 90 days. The primary outcome measures were changes in corneal staining according to the Oxford classification, results of the Schirmer test and tear break-up time (TBUT). The secondary outcome measures were changes in the Ocular Surface Disease Index (OSDI) and treatment compliance.

Results All subjects completed the study. The intervention group showed improvements in all primary outcome measures when compared with the control group, including a reduction in corneal staining $(p<0.001)$, increase in the mean Schirmer value from $5.6 \pm 0.7$ to $9.0 \pm 1.1 \mathrm{~mm}$, and an increase in TBUT from $4.0 \pm 0.4$ to $6.4 \pm 0.4 \mathrm{~s}$ at day 90 . An improvement in subjective OSDI values was also found.

Conclusion PRP injection is safe and effective in improving tear parameters as well as subjective parameters, and was found to be superior to hyaluronic acid alone in the management of patients with severe dry eye. This represent a novel alternative treatment for severe dry eye.
\end{abstract}

Trial registration number NCT02257957.

\section{INTRODUCTION}

Lacrimal gland dysfunction is the most common cause of severe dry eye and is secondary to several factors, including acinar atrophy, lacrimal gland fibrosis and ductal obstruction, as well as lymphocyte infiltration with upregulation of proinflammatory cytokines leading to a severe reduction in lacrimal gland secretion. The current treatments are palliative and are based on tear substitution, managing of tear retention and controlling ocular inflammation. 1

In the field of lacrimal regeneration, several approaches have been proposed: When the lacrimal gland is partially damaged with an insufficient number of cells, regeneration of the existing tissue has been proposed over regeneration using external tissue. This is because host cells are more suitable for regeneration than cells engineered by in vitro techniques. Based on this concept, novel procedures that involve the regeneration of existing cells must be developed. ${ }^{2}$

The use of platelet-rich plasma (PRP) has become a strategy in the management of several disorders of the ocular surface, including corneal ulcers ${ }^{3}$ and persistent ocular epithelial defects. ${ }^{4}$ PRP has an antiapoptotic effect on corneal stromal cells, ${ }^{5}$ and autologous platelets have been injected for the management of macular holes. ${ }^{67}$ Autologous platelets in the form of eye-drops or as a plasma gel have also been used in the management of several eye conditions depending on the desired effect and the purpose of the clinical intervention. In this way, PRP has great utility in the management of several ocular diseases. ${ }^{8}$

Injection is a common therapeutic approach when regeneration of determinate tissue is required. This approach is commonly used in sports medicine in the treatment of tendinopathy and management of knee osteoarthritis, and is supported by good evidence with high clinical significance. ${ }^{9}{ }^{10}$ It has also been used in the regeneration of several tissues due to the activity of several growth factors that can stimulate the proliferation and regeneration of stem cells, ${ }^{11}$ thereby increasing the number and stimulating the differentiation of cells required for tissue regeneration. Considering this rationale a potential new approach for treating patients with severe dry eye, this study proposes a novel strategy based on the concepts of regenerative medicine.

A case series of patients with severe dry eye in which PRP was injected into the lacrimal gland, resulting in an increase in lacrimal production and improvement of the symptoms of dry eye, has previously been described. ${ }^{12}$ Based on these observations, a prospective, randomised controlled study was developed to compare the efficacy of PRP injection with the current standard of care in order to determine if this treatment could be an option for patients with severe dry eye.

\section{MATERIALS AND METHODS \\ Patients}

Thirty patients from the Hospital de Engativa (Universidad Nacional de Colombia) were enrolled in the study. Patients were invited to participate if they had been diagnosed with Sjogren syndrome according to at least two of the American College of Rheumatology criteria $^{13}$ : (1) positive serum anti-Sjögren's-syndrome-related antigen $\mathrm{A}$ and/or 
anti--Sjögren's-syndrome-related antigen B or positive rheumatoid factor and antinuclear antibody titre $\geq 1: 320$, (2) ocular staining score of $\geq 3$, and /or (3) presence of focal lymphocytic sialadenitis with a foci score of $\geq 1$ focus per $4 \mathrm{~mm}^{2}$ in labial salivary gland biopsy samples.

Patients gave their informed consent to the procedure according to the Declaration of Helsinki, and the protocol was registered as a clinical trial under trial registration number NCT02257957.

Prior to the study, the purpose, possible adverse effects, limitations and benefits of the study were discussed with the patients. They were permitted to use artificial drops as required and were instructed to suspend the use of eye-drops on the day of the measurements. Patients did not undergo any additional procedures that could alter the lacrimal volume, such as lacrimal plugs or lacrimal occlusion, or received additional medications like ciclosporin or topical steroids. The exclusion criteria included patients who were pregnant, wore contract lenses, those with a systemic immune deficiency as AIDS, active allergy or active ocular infection, and those who used oral tetracyclines or omega III orally. The sample size was calculated based on our previous results, with an anticipated mean difference between groups of
$25 \%$, enrolment ratio of $1: 1$, type I/II error data with an alpha of 0.05 and a power of $95 \%$, giving a sample size of 11 patients per group. Accounting for a desertion rate of $30 \%$, it was determinated that 15 patients were required per group.

The 15 patients in the intervention group received a PRP injection at days $0,30,60$ and 90, while the 15 patients in the control group continued using only artificial tears (hyaluronic acid preservative-free) and were also evaluated at 0,30,60 and 90 days. Patients were randomised in a block design of size of 6 , for assignment into the groups. The allocation process also ensured that the physician who performed the injection was not the same as the one who examined (Schirmer, TBUT and staining) or questioned the patient for Ocular Surface Disease Index (OSDI) measurement. All patients received artificial tears as a part of dry eye management (figure 1).

\section{Autologous PRP preparation and injection}

For each patient on the PRP group, $10 \mathrm{~mL}$ of whole blood was collected by venipuncture into sterile tubes containing $0.5 \mathrm{~mL}$ sodium citrate (Becton Dickinson). The blood was centrifuged

\section{PRP injection in management of severe dry eye}

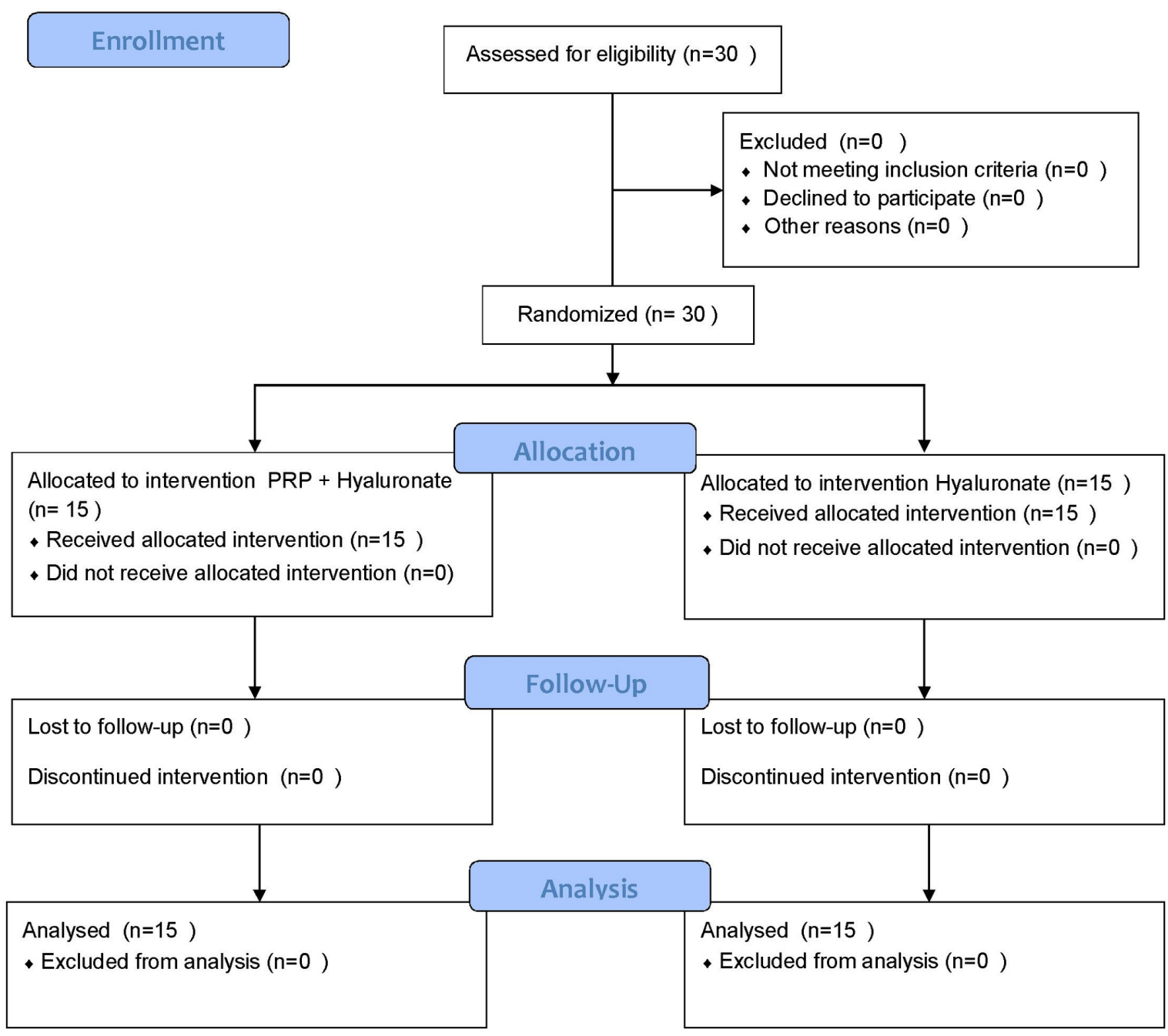

Figure 1 CONSORT flow diagram. CONSORT, Consolidated Standards of Reporting Trials; PRP, platelet-rich plasma. 


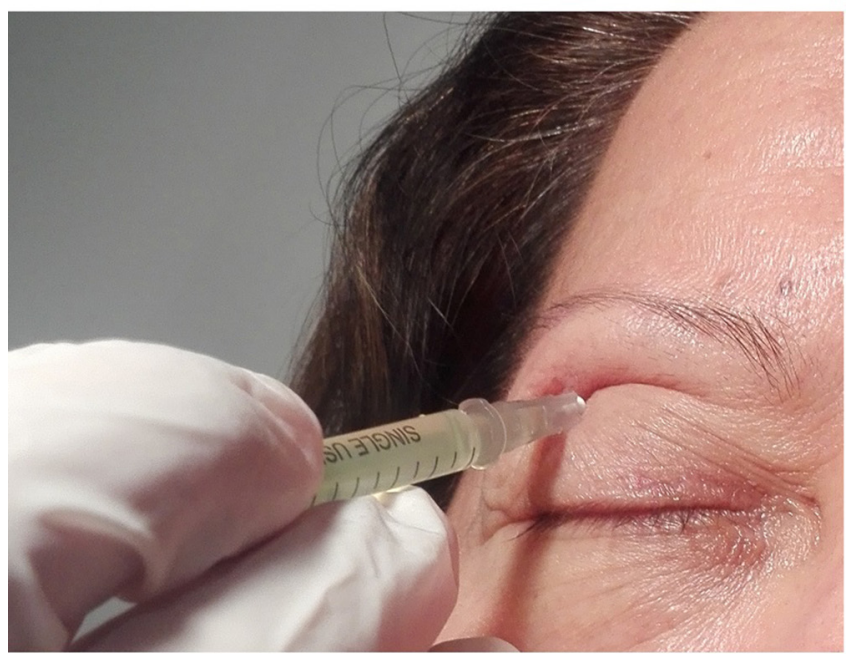

Figure 2 Platelet-rich plasma injection into the lacrimal gland transcutaneously. 1 cc is injected.

at $160 \mathrm{~g}$ for $10 \mathrm{~min}$. The plasma was isolated (approximately $4.5 \mathrm{~mL}$ per patient) and centrifuged again at $160 \mathrm{~g}$ for $15 \mathrm{~min}$. The bottom $1 \mathrm{~mL}$ was isolated and $0.1 \mathrm{~cm}^{3}$ of $\mathrm{CaCl}$ was added to activate the platelets. A $1 \mathrm{~mL}$ tuberculin syringe (Becton Dickinson) with a $27 \mathrm{G} \times 1 / 2$ inch needle was used for the injection. Approximately $4 \mathrm{~mm}$ of the needle was introduced into the orbital rim. The area to be injected had been previously disinfected with iodopovidone and isopropanol.

This fraction $(1 \mathrm{~mL})$ was injected transcutaneously into the external one-third of the orbital rim at a depth of $4 \mathrm{~mm}$ to the superior area. The reference was the area of the lacrimal fossa in the frontal bone. This anatomical area corresponds to the location of the lacrimal gland as previously described in procedures involving injection into the lacrimal gland ${ }^{14}$ (figure 2). This procedure was completed on days $0,30,60$ and 90 in both eyes of patients in the intervention group.

Postoperative care included observation of the area. There were no negative side effects, including erythema, oedema or severe pain, and analgesics that may alter platelet function were avoided.

Injections were performed by two surgeons (AMM and MYA) in order to avoid bias; the surgeon who performed the injection was not the same as the one who evaluated the patient.

\section{Clinical measurements}

Lacrimal volume

The Schirmer test I (performed without anaesthesia) was completed before any drops were instilled in the eye. Standardised Schirmer strips (Biotech Vision Care, Gandhinagar, India) were bent at the notch and placed carefully over the lower lid margin as far as possible towards the temporal angle of the lids. The patient was instructed to keep his/her eyelids closed during the test. The strips remained in place for $5 \mathrm{~min}$. After 5 min, the amount of moisture in each strip was measured using a millimetre scale.

\section{Tear break-up time}

Immediately after removal of the strips, a $20 \mu \mathrm{L}$ drop of $1 \%$ fluorescein was applied to the conjunctival fornix of each eye, then the participant was instructed to squeeze their eyelids tightly to remove excess dye. Immediately, cobalt blue was used in conjunction with an orange Wratten 12 filter. The time between blinking and appearance of the first dry spot was recorded as the tear break-up time (TBUT) and was measured three times using a chronometer and was averaged.

\section{Ocular surface staining}

A $1 \%$ solution of lissamine green was applied to the inferior conjunctiva. The staining was graded according to the Oxford grade panel and was classified from grade 0 to grade $\mathrm{V}^{7}$

\section{OSDI test}

The OSDI consists of 12 questions on items related to the assessment of symptoms, functional limitations and environmental factors related to dry eye. It is graded on a scale of $0-4$, with 0 indicating none of the time, 1 for some of the time, 2 for half of the time, 3 for most of the time and 4 indicating all the time. The total OSDI score was then calculated by the following formula: OSDI $=$ (sum of scores for all questions answered) $\times 100 /($ total number of questions answered) $\times 4$ ). A score of $0-100$ was obtained, related to the extent of dry eye. This is a valid and reliable instrument for measuring the severity of dry eye. ${ }^{8}$

\section{Statistical analysis}

The results are presented as the mean \pm SEM. Normality was determined using the Shapiro-Wilk test. Significance was evaluated by comparing both groups (control vs treated, or pretreatment vs post-treatment) of two sets of data using a paired Student's t-test. Groups of three or more measurements (baseline, 30, 60 and 90 days) were evaluated by one-way or two-way analysis of variance (ANOVA), as appropriate, followed by the Bonferroni post-hoc test. Differences were considered significant at $\mathrm{p}<0.05$.

\section{RESULTS}

All subjects completed the study, with none dropping out. The mean age of patients was $59.2 \pm 3.4$ years in the PRP treatment group and $52.7 \pm 3.5$ years in the control group. The baseline values were similar between groups (table 1). The injection was well tolerated in the treatment group, and there were no adverse effects. Some patients presented with temporary mild oedema postinjection.

\section{Lacrimal volume}

The lacrimal volume was $5.4 \pm 0.7 \mathrm{~mm}$ in the PRP group at baseline vs $5.6 \pm 0.40 \mathrm{~mm}$ in the control group at baseline.

Table 1 Demographics of patients

\begin{tabular}{lllllll}
\hline Treatment & Age & Gender & Biopsy & Diagnosis SSB+ & SSA+ & Staining \\
\hline Platelet-rich plasma injection & $59.2 \pm 3.5$ & 14 female, 1 male & 9 & 13 & 12 & 15 \\
Control & $52.7 \pm 3.5$ & 14 female, 1 male & 11 & 14 & 10 & 15 \\
\hline
\end{tabular}

Patients were diagnosed with Sjogren syndrome according to the American College of Rheumatology criteria. Biopsy with focal sialadenitis with focus score higher than 1 focus per $\mathrm{mm}^{2}$, anti-SSB and anti-SSA higher than $1 / 320$, and ocular staining $\geq 4$ according to the van Bijsterveld classification.

SSA, Sjögren's-syndrome-related antigen A; SSB, Sjögren's-syndrome-related antigen B. 


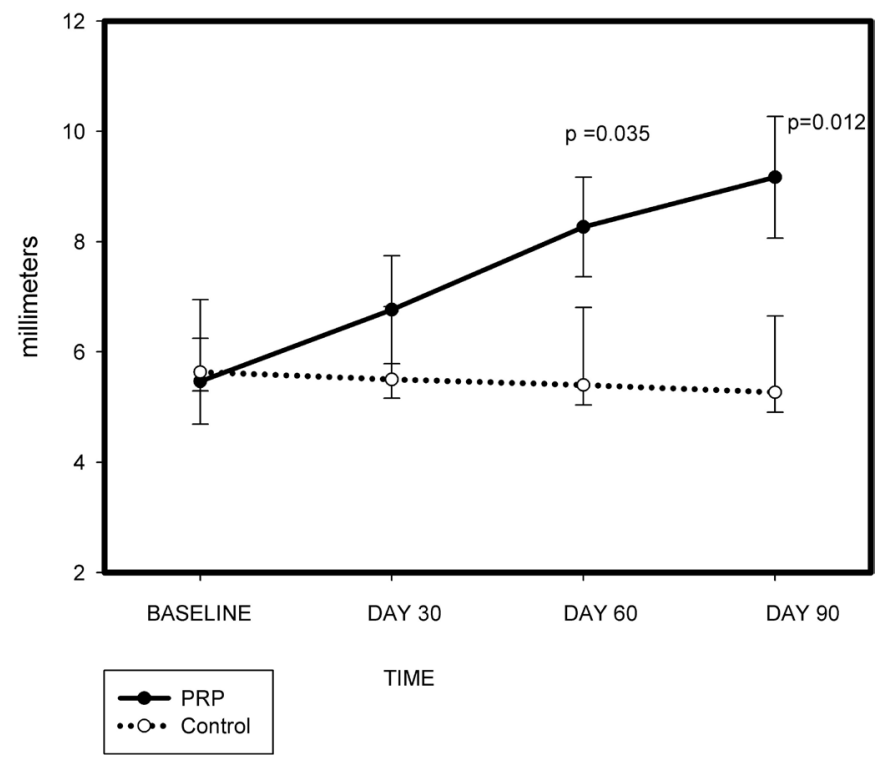

Figure 3 Schirmer values at baseline and at 30,60 and 90 days. Solid line indicates PRP injection treatment, and dotted line denotes the control group. Values are in millimetres, and significant differences (paired t-test $\mathrm{p}<0.05$ ) between the two groups were observed at days 60 and 90. PRP, platelet-rich plasma.

Lacrimal production increased from the first to the last visit in the PRP group (from $6.7 \pm 0.9 \mathrm{~mm}$ to $9.2 \pm 1.0 \mathrm{~mm}$ ) but not in the control group (from $5.50 \pm 0.5 \mathrm{~mm}$ to $5.3 \pm 0.7 \mathrm{~mm}$ ). This difference was significant, according to ANOVA $(\mathrm{p}<0.002)$ (figure 3).

\section{Tear break-up time}

The TBUT at baseline was $4.0 \pm 0.4 \mathrm{~s}$ in the intervention group and $4.2 \pm 0.3 \mathrm{~s}$ in the control group, and this difference was not significant $(p=0.655)$. At the fourth visit, the values were

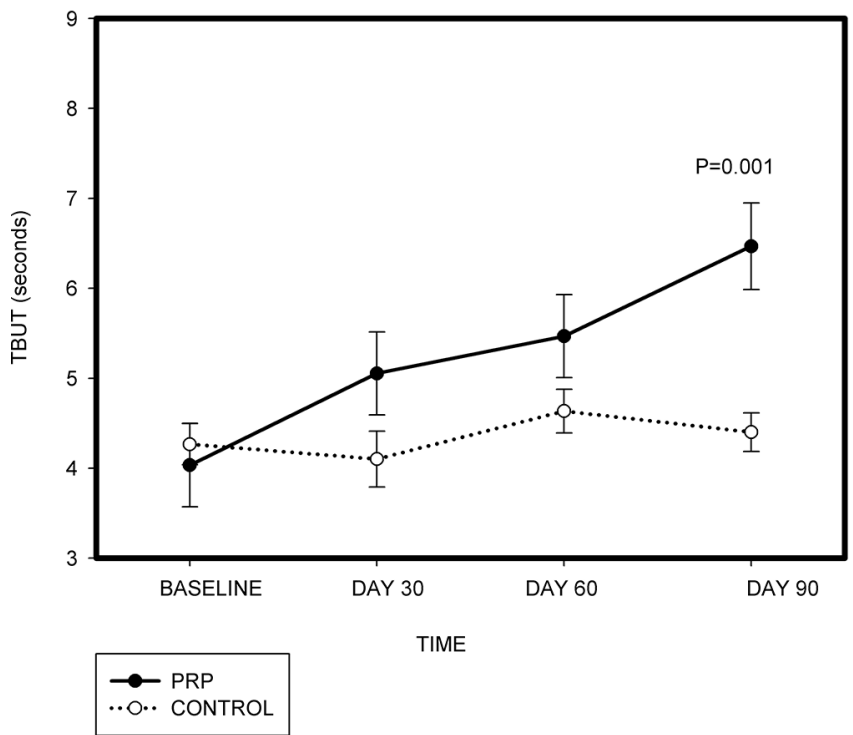

Figure 4 TBUT in seconds at baseline and at 30,60 and 90 days. Solid line indicates PRP injection treatment, and dotted line denotes the control group. Significant differences (paired t-test $p<0.05$ ) between the two groups were observed at day 90. PRP, platelet-rich plasma; TBUT, tear break-up time.
$6.4 \pm 0.4 \mathrm{~s}$ in the PRP group vs $4.4 \pm 0.3 \mathrm{~s}$ in the control group. This was a significant improvement $(p=0.0055)$ (figure 4$)$.

\section{Ocular surface staining}

The values for ocular surface staining at baseline were $2.5 \pm 0.15$ in the PRP treatment group and 2.6 \pm 0.16 in the control group. These values were reduced to $1.2 \pm 0.18$ in the treatment group and $2.4 \pm 0.18$ in the control group at day 90 , and this difference between groups was significant (paired t-test pretreatment vs post-treatment, $\mathrm{p}<0.001$, t-value 6.09) (figure 5A).

\section{OSDI score}

The treatment and control groups had similar OSDI scores at baseline, with no significant difference between groups. At 90 days, the OSDI value of the treatment group decreased from $59 \pm 4.0$ to $34 \pm 4.0$ and from $54 \pm 3.5$ to $55 \pm 2.5$ in the control group (paired t-test $\mathrm{p}<0.001$ ) (figure $5 \mathrm{~B}$ ).

\section{DISCUSSION}

In this study PRP treatment was associated with an improvement in all evaluated parameters of lacrimal function, characterised by an increase in lacrimal volume. Indeed we observed an increase of approximately $64 \%$ from baseline in patients with Sjogren syndrome with severe lacrimal alteration. Despite the variability in the Schirmer index reported by several studies, it is still considered valid and has been approved in several populations for the diagnosis, study and follow-up of patients with Sjogren syndrome.

We observed a significant improvement in TBUT values in the PRP group, with an increase from baseline values in $45 \%$ of the PRP group. This improvement suggests that PRP treatment increases the stability of the lacrimal film. The increase in lacrimal volume combined with the improvement in tear components of the tear film may reflect an improvement in the volume and in the quality of tear components. This may also reflect an improvement in meibomian gland function associated with paracrine regulation.

The values obtained for conjunctival and corneal staining values were decreased due to the PRP injection. This change was observed from the 60th day onwards and was more evident on 90 days post-treatment $(\mathrm{p}=0.001)$, indicating an improvement in all lacrimal proteins and lipids and reflecting an improvement in ocular surface health.

An improvement in the symptom score measured using the OSDI was found in the treated group, with an 18-point reduction from baseline. This score is an important indicator of patient functionality and rehabilitation. While the average score in this group was still within the range of severe dry eye (from 33 to 100), patients reported that they were able to perform activities such as reading and outdoor activities with more comfort than before they had received the treatment.

PRP contains several components with known proregenerative capabilities, and in vivo studies have demonstrated its ability to improve cellular recruitment of dermal-derived stem cells with the formation of sweat glands as well as acinar and ductal cells, regenerating a dermis-like tissue. ${ }^{15}$ It has also been shown to have an effect on the preservation of stem cell properties in several tissues. ${ }^{16}$ In addition PRP has been reported to positively affect the glandular cells of the liver by a direct effect on hepatocytes, as well as an indirect antifibrotic effect and a protective effect against hepatocyte apoptosis. ${ }^{17}$ PRP also contains several growth factors and cytokines that have shown 


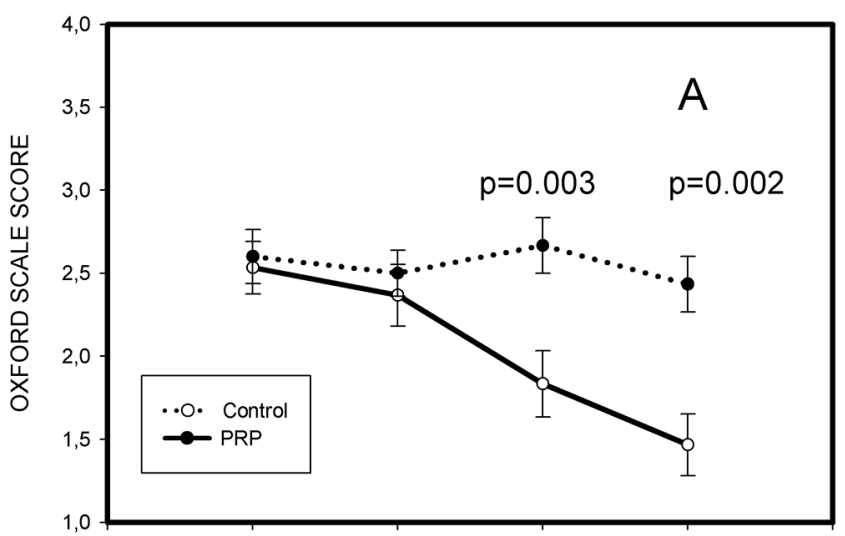

BASELINE DAY 30 DAY 60 DAY 90

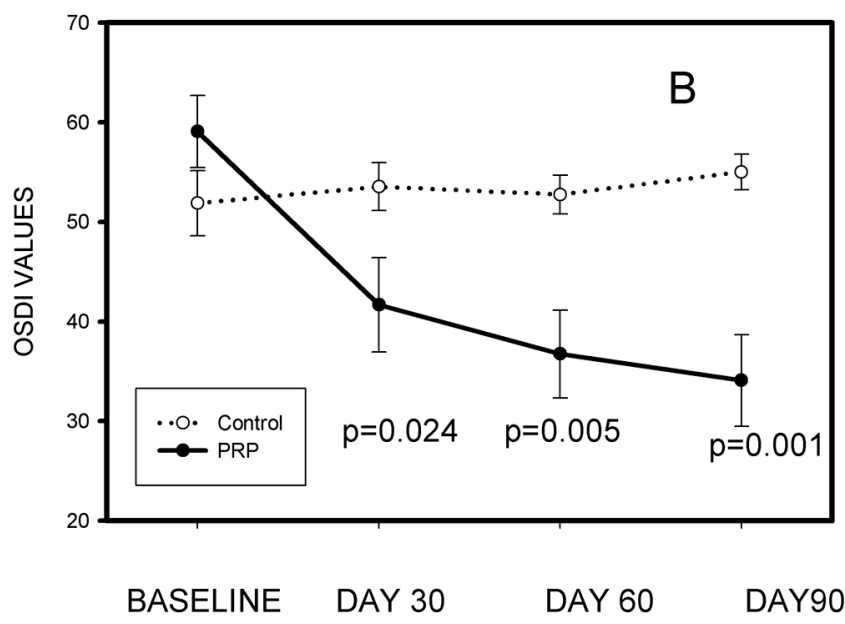

Figure 5 (A) Ocular surface staining values according to the Oxford classification at baseline and at 30,60 and 90 days. Solid line indicates PRP injection treatment, and dotted line denotes the control group. Significant differences (paired t-test $p<0.05$ ) between the two groups were observed at days 60 and 90. (B) OSDI symptom values at baseline and at 30, 60 and 90 days. Solid line indicates PRP injection treatment, and dotted line denotes the control group. Significant differences (paired t-test $\mathrm{p}<0.05$ ) between the two groups were observed at days 60 and 90. OSDI, Ocular Surface Disease Index; PRP, platelet-rich plasma.

to promote the regeneration of secretory tissues both in vitro and in vivo. ${ }^{18}$

Our group has previously reported an effect of PRP in patients with severe dry eye. In the current study, we found an effect on subjective and objective measurements in this set of patients. This approach is a novel way to improve function, as a palliative treatment for dry eye and as a regenerative approach.

Until now, therapies have focused on reducing lacrimal gland inflammation, increasing tear flow production and increasing retention therapy; however, none of these approaches can regenerate the lacrimal gland.

In order to achieve in situ lacrimal regeneration, complete restoration of the original tissue must occur. Ideally, any regenerative therapy must restore tear volume, have anti-inflammatory properties, be non-immunogenic (autologous is ideal), comply with Good Manufacturing Practice, be cost-effective (dry eye syndrome is highly prevalent) and have no adverse side effects. ${ }^{2}$

The results of this study demonstrate that PRP injection fulfils several of these criteria, including increased tear volume, being non-immunogenic and autologous, cost-effective, and having no adverse side effects. Moreover, patients' symptoms were improved following use.

The use of PRP for ocular therapies has gained attention, and several kits have been developed for this purpose. These kits (eg, Endoret BTI, COL System Biomed) optimise the yield and quality of PRP, and systems like these should be considered for development specifically for injection in order to increase the safety of this approach.

\section{CONCLUSIONS}

PRP injection is a safe and effective treatment for improving lacrimal function and is feasible for patients with severe dry eye syndrome. In our study the injections were performed on days $0,30,60$ and 90 based on the schedule usually performed by dermatologists in hair regeneration. Future studies should investigate the use of isolated growth factors and, potentially, autologous stems cells. Our study reports the first step in a practical approach for the regeneration of lacrimal function.

Contributors Contribution of each author is listed as the following. MYA, AMM, AMl: substantial contribution to the conception or design of the work, or the acquisition, analysis or interpretation of data. MYA, AMA: drafting the work or revising it critically for important intellectual content. MYA, AMM, AMI: final approval of the version published. MYA, AMM, AMI: agreement to be accountable for all aspects of the work in ensuring that questions related to the acuity or integrity of any part of the work are appropriately investigated and resolved.

Funding This research was funded by Facultad de Medicina Convocatoria de trabajos de grado 2016.

Competing interests None declared.

Patient consent Obtained.

Ethics approval The protocol was approved by Comite de Etica Facultad de Medicina Universidad Nacional de Colombia.

Provenance and peer review Not commissioned; internally peer reviewed.

\section{REFERENCES}

1 Jones L, Downie LE, Korb D, et al. TFOS DEWS II management and therapy report. Ocul Surf 2017;15:575-628.

2 Dietrich J, Massie I, Roth M, et al. Development of causative treatment strategies for lacrimal gland insufficiency by tissue engineering and cell therapy. Part 1: Regeneration of lacrimal gland tissue: Can we stimulate lacrimal gland renewal in vivo? Curr Eye Res 2016;41:1131-42.

3 Alio JL, Rodriguez AE, WróbelDudzińska D. Eye platelet-rich plasma in the treatment of ocular surface disorders. Curr Opin Ophthalmol 2015;26:325-32.

4 Ronci C, Ferraro AS, Lanti A, et al. Platelet-rich plasma as treatment for persistent ocular epithelial defects. Transfus Apher Sci 2015;52:300-4.

5 Koulikovska M, Szymanowski O, Lagali N, et al. Platelet-rich plasma prolongs myofibroblast accumulation in corneal stroma with incisional wound. Curr Eye Res 2015:40:1102-10.

6 Konstantinidis A, Hero M, Nanos P, et al. Efficacy of autologous platelets in macular hole surgery. Clin Ophthalmol 2013;7:745-50.

7 Engelmann K, Sievert U, Hölig K, et al. [Effect of autologous platelet concentrates on the anatomical and functional outcome of late stage macular hole surgery: A retrospective analysis]. Bundesgesundheitsblatt Gesundheitsforschung Gesundheitsschutz 2015;58(11-12):1289-98. 1.

8 Alio JL, Arnalich-Montiel F, Rodriguez AE. The role of "eye platelet rich plasma" (E-PRP) for wound healing in ophthalmology. Curr Pharm Biotechnol 2012;13:1257-65.

9 Fitzpatrick J, Bulsara M, Zheng MH. The effectiveness of platelet-rich plasma in the treatment of tendinopathy: A Meta-analysis of Randomized Controlled Clinical Trials. Am J Sports Med 2017;45:226-33.

10 Meheux CJ, McCulloch PC, Lintner DM, et al. Efficacy of intra-articular plateletrich plasma injections in knee osteoarthritis: A systematic review. Arthroscopy 2016;32:495-505. 
11 Tobita M, Tajima S, Mizuno H. Adipose tissue-derived mesenchymal stem cells and platelet-rich plasma: stem cell transplantation methods that enhance stemness. Stem Cell Res Ther 2015;6:215.

12 Avila MY. Restoration of human lacrimal function following platelet-rich plasma injection. Cornea 2014;33:18-21.

13 Shiboski SC, Shiboski CH, Criswell L, et al. American College of Rheumatology classification criteria for Sjögren's syndrome: a data-driven, expert consensus approach in the Sjögren's International Collaborative Clinical Alliance cohort. Arthritis Care Res 2012;64:475-87.

14 Montoya FJ, Riddell CE, Caesar R, et al. Treatment of gustatory hyperlacrimation (crocodile tears) with injection of botulinum toxin into the lacrimal gland. Eye 2002;16:705-9.
15 Roubelakis MG, Trohatou O, Roubelakis A, et al. Platelet-rich plasma (PRP) promotes fetal mesenchymal stem/stromal cell migration and wound healing process. Stem Cell Rev 2014;10:417-28.

16 Anitua E, Prado R, Orive G. Safety and efficient ex vivo expansion of stem cells using platelet-rich plasma technology. Ther Deliv 2013;4:1163-77.

17 Matsuo R, Nakano Y, Ohkohchi N. Platelet administration via the portal vein promotes liver regeneration in rats after $70 \%$ hepatectomy. Ann Surg 2011;253:759-63.

18 Ross J, Gherardi E, Mallorqui-Fernandez N, et al. Protein engineered variants of hepatocyte growth factor/scatter factor promote proliferation of primary human hepatocytes and in rodent liver. Gastroenterology 2012;142:897-906. 